\title{
Appendix to Visualizing Complex Data with Embedded Plots
}

\author{
Garrett Grolemund* \\ RStudio \\ and \\ Hadley Wickham ${ }^{\dagger}$ \\ Department of Statistics, Rice University
}

December 23, 2013

\section{A ADVANCED IMPLEMENTATION}

The layered grammar of graphics includes more than just geoms and aesthetic mappings. It also uses stats, parameters, position adjustments, and coordinate axes to describe and build graphs (Wickham, 2010). This appendix discusses how each of these can be used to create a useful interface for building embedded plots. We illustrate our ideas with code from the ggsubplot package.

\section{A.1 Geoms}

Software that creates embedded plots should provide a quick way to build glyph-like geoms. Glyphlike geoms can be built in current implementations of the grammar of graphics by combining traditional geoms with polar coordinates. For example, an analyst can build a star geom by combining a line geom with polar coordinates, Figure 1.a, or a frequency polygon geom with polar coordinates, Figure 1.b. He or she can build a coxcomb geom by combining a bar geom with polar coordinates, Figure 1.c.

\footnotetext{
*Garrett Grolemund is Statistician, RStudio, Boston, Massachusetts 02210 (email: grolemund@rstudio.com)

${ }^{\dagger}$ Hadley Wickham is Adjunct Professor, Rice University, Houston, TX 77005 (email: hadley@rice.com)
} 


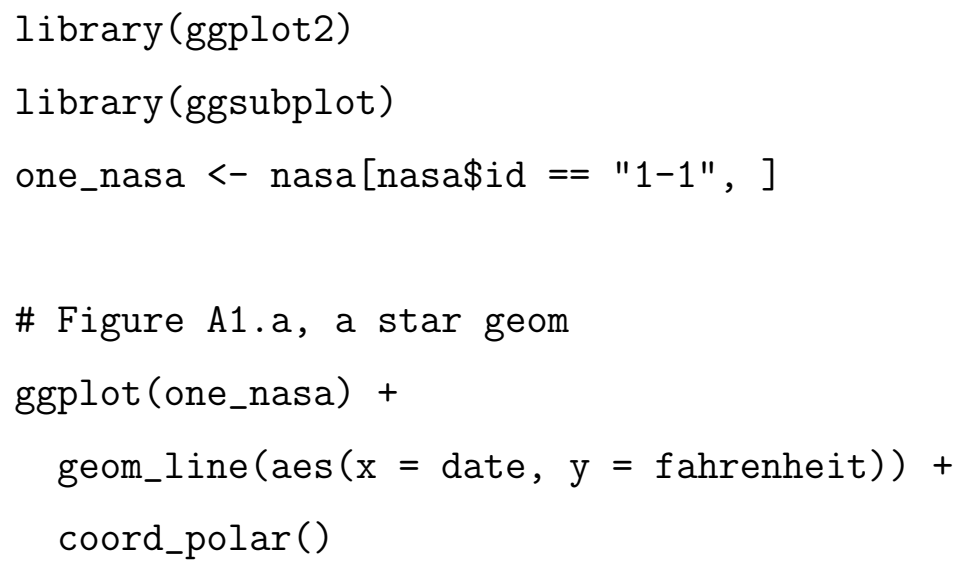

This two step process is fine for traditional plots, where glyph-like geoms are rarely used. Embedded plots, however, use glyph-like subplots frequently. These subplots will be easier to create if glyph-like objects are already available as pre-made geoms. For example, an analyst can use ggsubplot to draw a star, Figure 1.d; freqstar, Figure 1.e; or coxcomb geom, Figure 1.f. Each is called with a standard geom syntax like below and is analogous to the polar version.

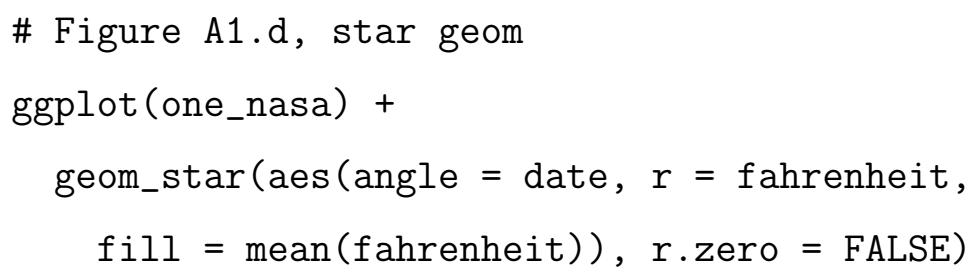


geom_freqstar (aes (angle = ozone, fill = mean(ozone))

\# Figure A1.f, coxcomb geom
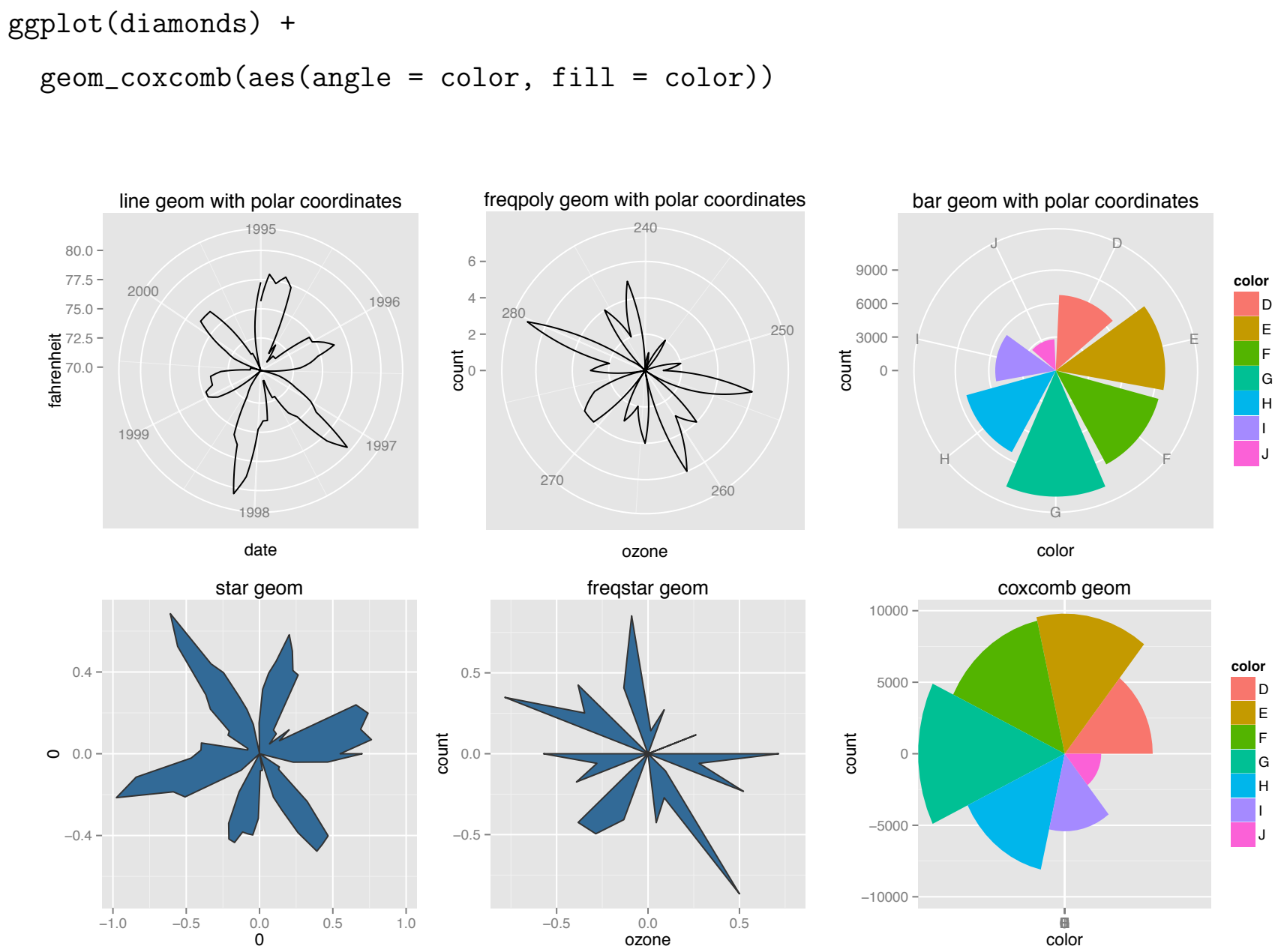

Figure 1: In the layered grammar of graphics, glyph-like objects can be built by combining common geoms with polar coordinates (A, B, C - top row). However, this arrangement can be cumbersome when building subplots. In that case, it is more convenient to create glyph-like objects with their own geoms (D, E, F - bottom row)

\section{A.2 Mapping and Stats}

Aesthetic mappings for subplots must behave differently than aesthetic mappings for simple geoms. When you place a simple geom, like a point, in the coordinate plane, you map a single data value 
to a single x coordinate, and a single data value to a single y coordinate, Figure 2.a. Each subplot, however, describes a group of points. To place a subplot in the $\mathrm{x}$ and $\mathrm{y}$ coordinate field, you must map a group of data values to a single $\mathrm{x}$ value and a group of data values to a single y value, Figure 2.b. This can easily be done by taking the max, min, mean, median, mode, etc. of the group of values.

\section{Mapping simple geoms}

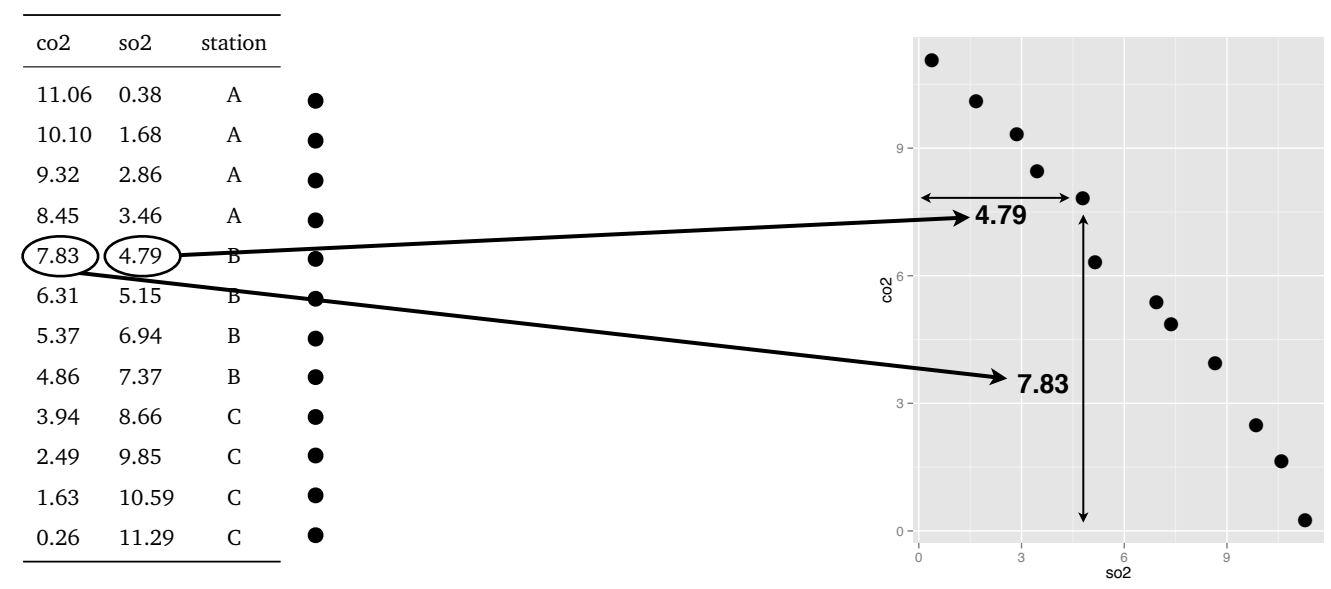

Mapping subplots

\begin{tabular}{llc}
\hline co2 & so2 & station \\
\hline 11.06 & 0.38 & $\mathrm{~A}$ \\
10.10 & 1.68 & $\mathrm{~A}$ \\
9.32 & 2.86 & $\mathrm{~A}$ \\
8.45 & 3.46 & $\mathrm{~A}$ \\
7.83 & 4.79 & $\mathrm{~B}$ \\
6.31 & 5.15 & $\mathrm{~B}$ \\
5.37 & 6.94 & $\mathrm{~B}$ \\
4.86 & 7.37 & $\mathrm{~B}$ \\
3.94 & 8.66 & $\mathrm{C}$ \\
2.49 & 9.85 & $\mathrm{C}$ \\
1.63 & 10.59 & $\mathrm{C}$ \\
0.26 & 11.29 & $\mathrm{C}$ \\
\hline
\end{tabular}

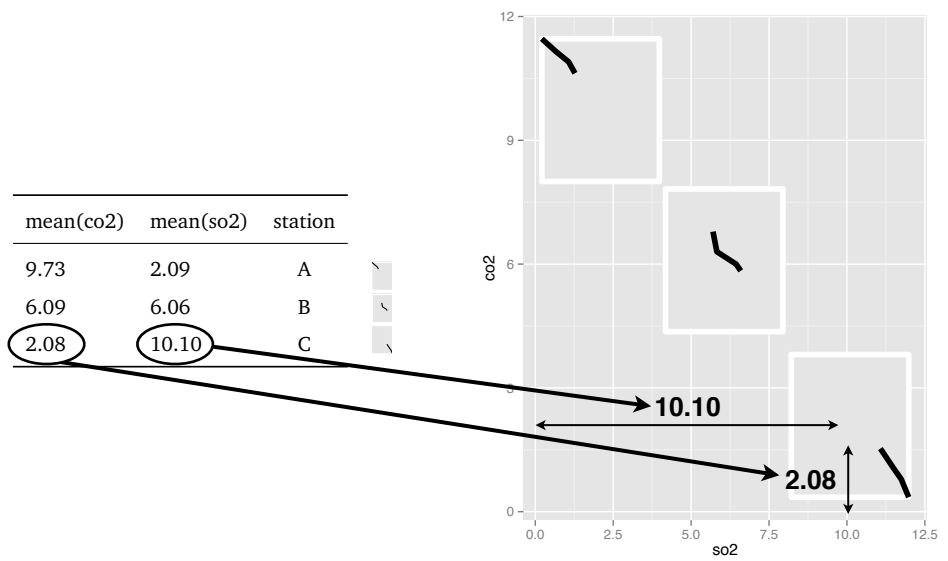

Figure 2: Simple geoms map one point to one object. A subplot maps a group of points to one subplot. To place a subplot, a user must map a group of values to a single $\mathrm{x}$ or y point. One way to do this intermediate transformation is with a stat.

In the layered grammar of graphics, such group-wise summaries are done with a stat. A stat 
is a specific transformation that transforms a user's data set into a new data set that can be used to draw geoms. For example, in ggplot2, the boxplot geom uses a stat. Boxplots behave like subplots, they describe multiple data points with a single geom object. To place a boxplot, ggplot2 first uses a stat to transform the group of values to a set of five numbers (a median, a $25^{\text {th }}$ percentile, a $75^{\text {th }}$ percentile, etc.). These numbers are then used to draw the boxplot. The histogram geom does something similar. It uses a stat to transform a group of values into a set of bins and counts. ggplot2 then uses these bins and counts to draw the histogram. In ggplot2 stats come pre-packaged with names like stat_boxplot, stat_bin, and stat_quantile. Every geom is associated with a default stat, so users usually do not need to worry about transforming their data with a stat to build their graph; ggplot2 takes care of it automatically.

A programmer can add a stat that positions subplots to their software. However, a user would then be limited to positioning subplots with the transformations provided by the stat. In ggsubplot, we demonstrate an alternative approach. Mappings for subplots are automatically calculated on a group-wise basis. If a user passes a mapping such as $\mathrm{x}=$ mean (surftemp), ggsubplot does not use the entire data set to calculate mean (surftemp) (which would result in a single value). ggsubplot first divides the data set into groups according to the subplot geom's group mapping. Then ggsubplot calculates mean (surftemp) once for each group, which results in a separate value for each subplot. This procedure is analogous to the split-apply-combine strategy described by Wickham (2011).

This arrangement offers two advantages over supplying the user with a stat. First, the user can use any function they wish to position subplots; the user is not limited to transformations that exist as a stat. The only constraint is that the user must choose a function that takes a group of values and returns a single value. Second, the method can be abstracted to use with nonembedded plots. For example ggsubplot provides the ply_aes function, which takes a ggplot2 layer object and modifies it so the layer's mappings are calculated by group according to the layer's group aesthetic. ply_aes enforces summarization by subsetting the output of each mapping to just its first value. A warning message is given if the mapping would have otherwise returned multiple values. geom_subplot automatically uses ply_aes. Figure 3 shows how this technique can remarkably reduce overplotting to reveal structure.

\section{\# Figure A3.a overplotted ozone vs. temperature}



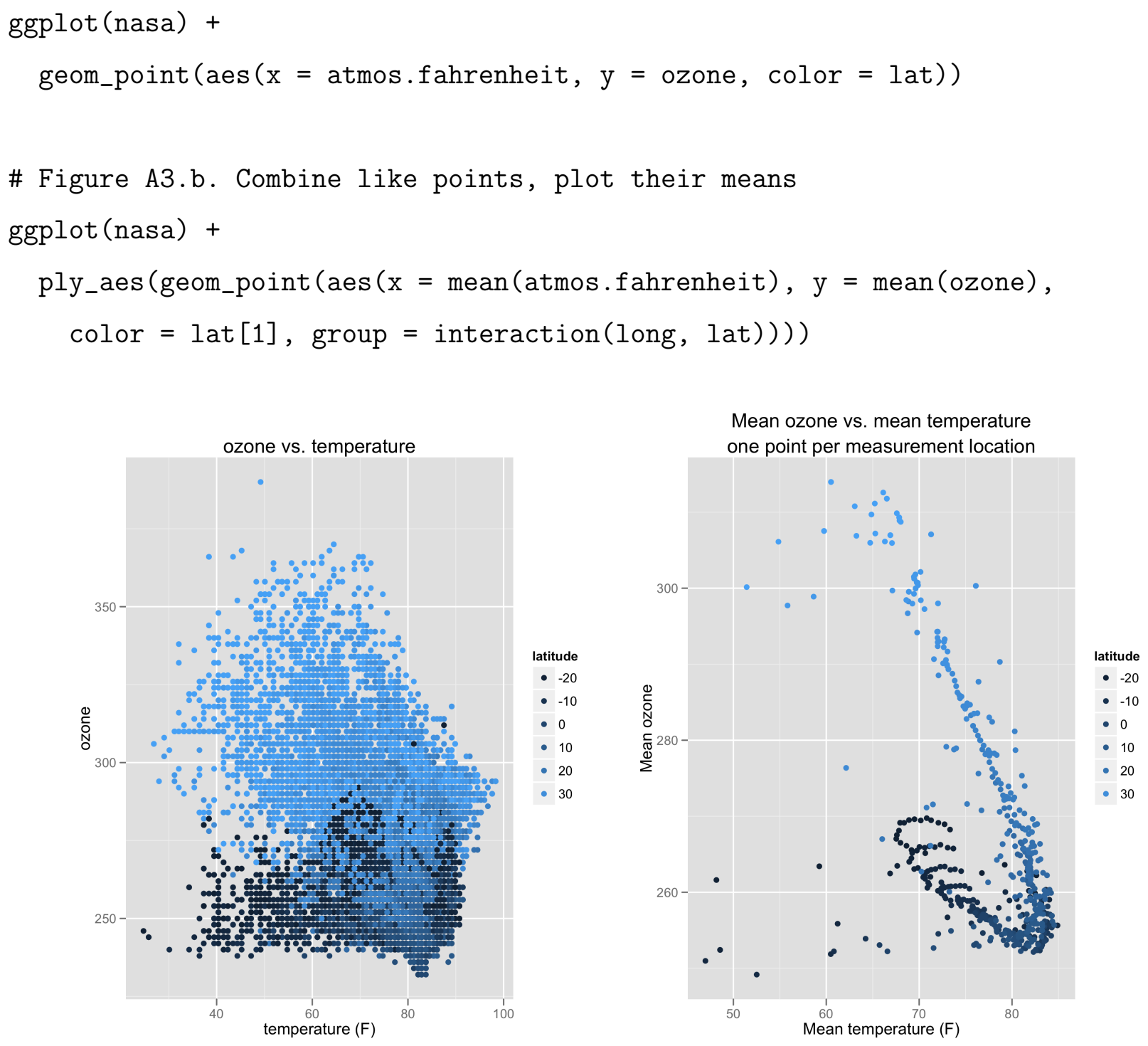

Figure 3: ply_aes offers a new strategy for overplotted graphs, like the one on the left. Groups of geoms are combined into single geoms that display summary information. This approach reveals that mean(ozone) has a different linear relationship with temperature in the southern hemisphere than it does in the northern hemisphere (right). Each collection of points that share the same latitude and longitude on the left is represented by a single point on the right.

\section{A.3 Parameters}

The layered grammar of graphics allows users to control the visual appearance of geoms with parameters. Both parameters and aesthetic mappings can influence visual aspects of a geom, such 
as size, shape, and color. However, parameters and mappings influence the geom in different ways. An aesthetic mapping will use values in the data set to choose the specific size, shape, and color of a specific geom. As a result, variations in the size, shape, and color will mirror variations in the values of the data set. In contrast, parameters set the size, shape, and colors of every geom in a layer to the same user-supplied value. In other words, parameters let the user manually tweak the appearance of geoms in a plot.

The two most useful parts of a subplot to tweak are the width and height of the subplot. ggsubplot allows users to manually adjust these dimensions with width and height parameters, Figure 4. Each is measured in the units of the respective major $\mathrm{x}$ (or y) axis.

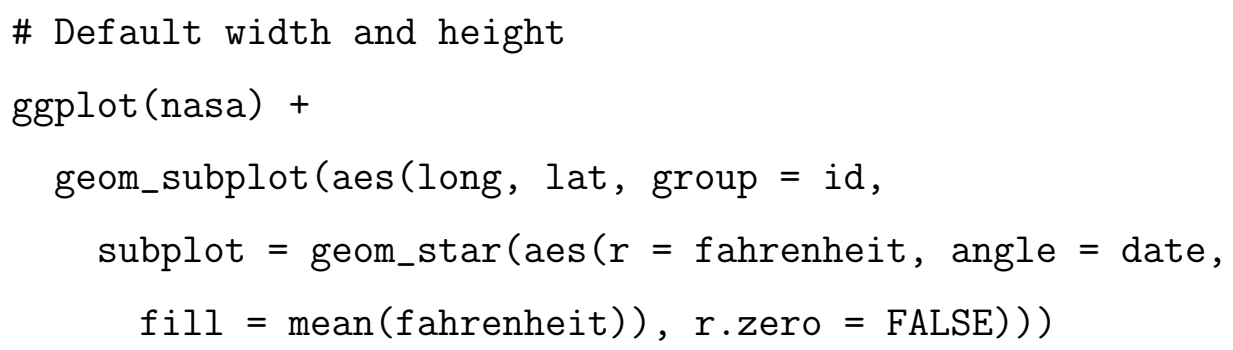

\# Decreased width and height

ggplot (nasa) +

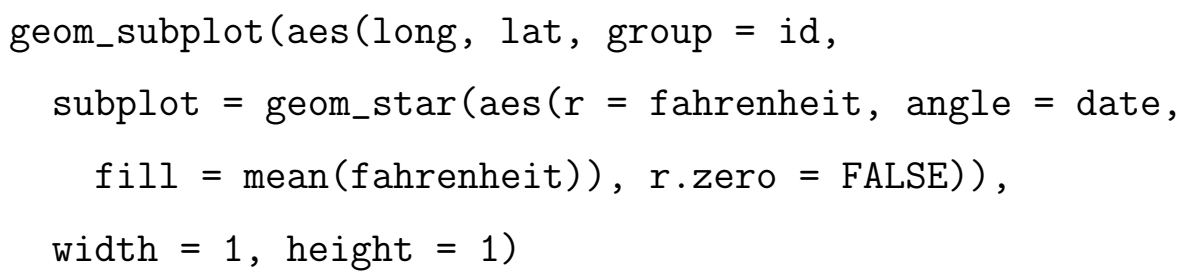

ggsubplot also provides a relational system that can simplify setting width and height. ggsubplot identifies the smallest distance between any two subplots on the $\mathrm{x}$ axis and uses this as the default width. ggsubplot identifies the smallest distance between any two subplots on the $\mathrm{y}$ axis and uses this as the default height. A user can set the height or width to a proportion of these defaults with the rel function, Figure 4.

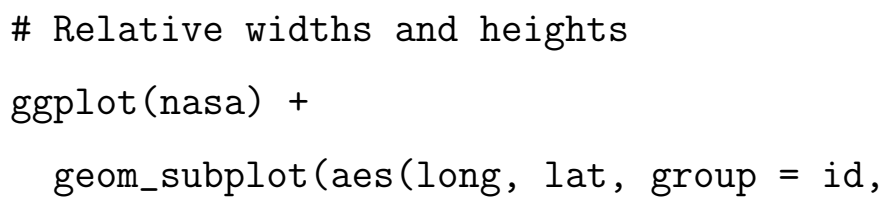




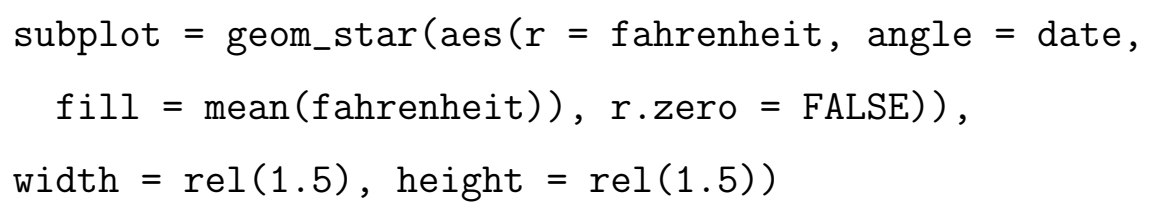

Glyphs with default width and heights

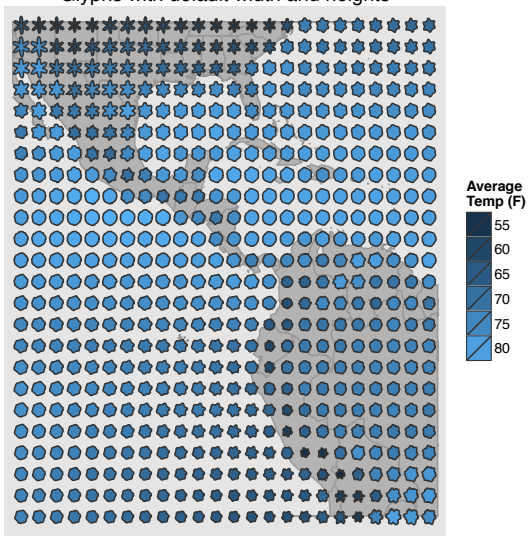

Glyphs with decreased width and heights

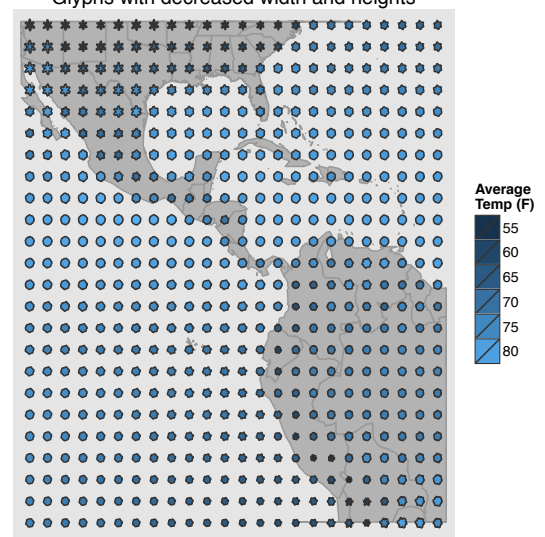

Increased width and heights, set with rel()

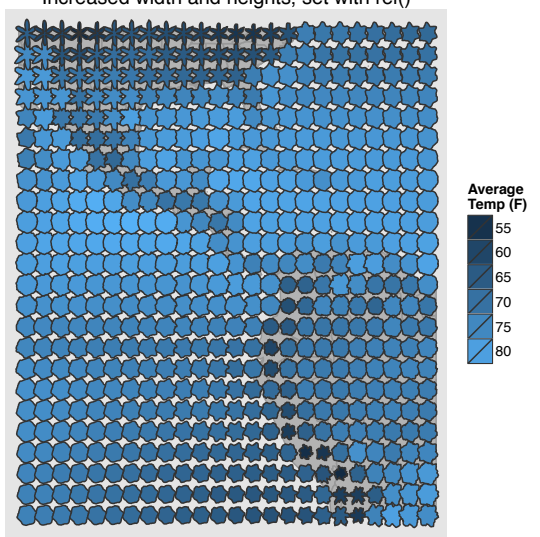

Figure 4: A user can control the width and height of subplots with the width and height parameters. Width is measured in the units of the major $\mathrm{x}$ axis, height in the units of the major y axis. Alternatively, a user can use the rel function to set width and height to proportions of the default width and height. The plot on the right uses a width and height equal to 1.5 times the default width and height. This is accomplished by setting width $=\operatorname{rel}(1.5)$, height $=\operatorname{rel}(1,5)$.

\section{A.4 Position adjustments}

The geoms in a plot will often overlap, or "collide", with each other. In this case, a user may wish to alter the positions of the geoms so that they do not interfere with each other. In the layered grammar of graphics, each layer of a graphic contains a position adjustment that determines how to plot graphical elements that interfere with one another. Wilkinson and Wills (2005) refer to this concept as a collision modifier. The ggplot2 implementation of the grammar of graphics contains four possible position adjustments. Overlapping elements can be adjusted to appear above each other (stacking), next to each other (dodging), in random nearby locations (jittering), or left as they are to overlap (identity).

A user may feel that these arrangements do not work well for embedded plots, because subplots often use position in a special way. The location of a subplot signals which data points have been 
included in the subplot. Adjusting the location of the subplot would disrupt this relationship.

A programmer can attempt to handle overplotting without moving subplots by merging overlapping subplots into a single subplot. However, this tactic presents difficult programming challenges: software must identify which subplots overlap, decide how many subplots should be used to display a cluster of overlapping subplots, and determine the size and the location of merged subplots. ggsubplot provides the merge position adjustment, which implements this approach with limited success, Figure 5.b.

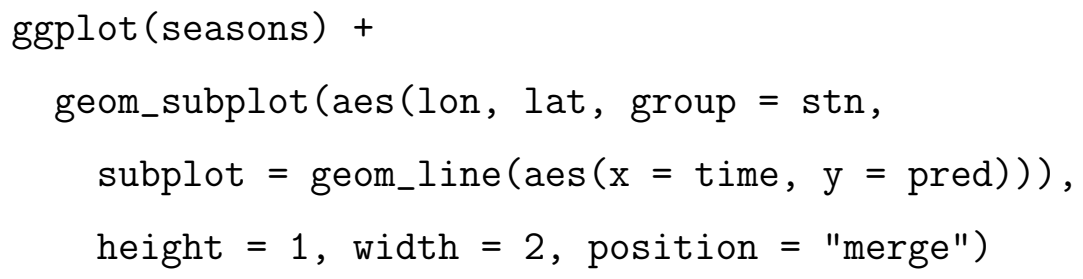

The same problem can be solved in a far simpler manner, by first binning the data into a $2 \mathrm{D}$ grid, and then drawing a separate subplot for each bin in the grid, Figure 5.c. ggsubpot provides a specific geom, geom_subplot2d, to do just this.

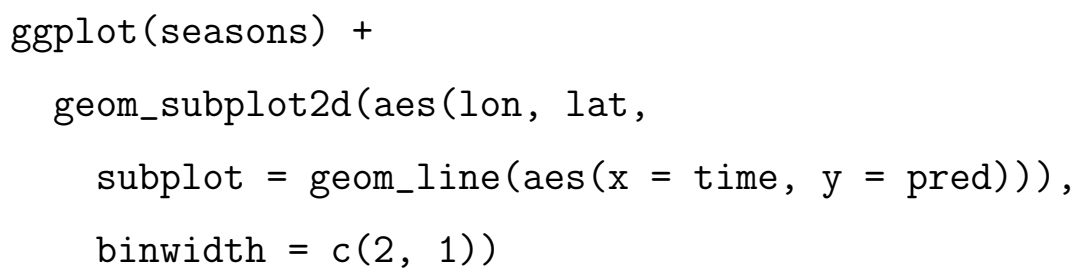

\section{A.5 Reference objects}

Graphs usually come with a set of coordinate axes that act as a reference object for the plot. Users can judge values by scanning the axes, and users can use the axes to make comparisons across multiple plots. However, axes are difficult to read at the small scales used in subplots. Boxes and lines can also allow comparison and scale better to the smaller sizes of subplots. ggsubplot creates these objects with a reference parameter in the subplot layer, see Figure 6.

These reference objects allow viewers to judge the position of geoms inside the subplot and to make comparisons against the position of geoms in other subplots. The dimensions of reference objects do not vary across subplots, which facilitates accurate comparisons. However, other features 

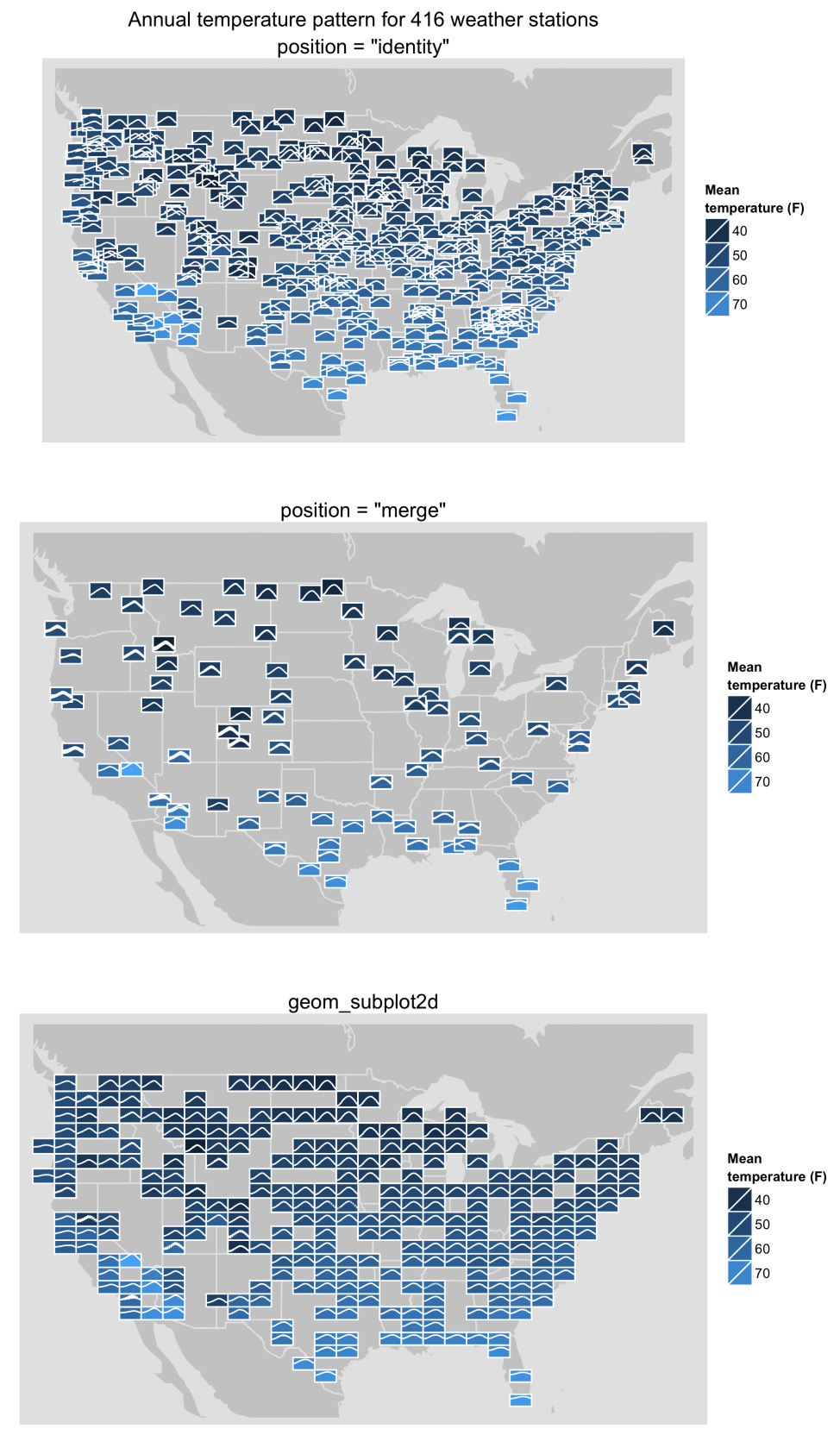

Figure 5: Temperature changes from 2000 to 2001 for multiple locations. The position of a subplot is often related to which points the subplot shows. Position = merge and geom_subplot2d provide two ways to avoid overlapping subplots without disrupting this relationship. 

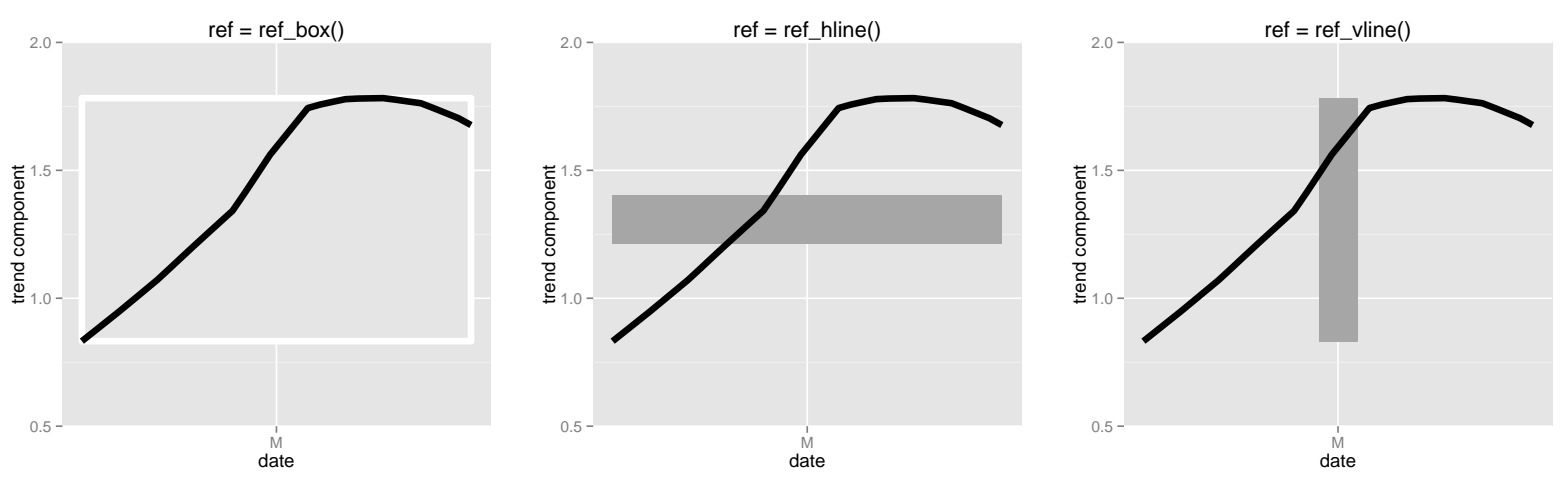

Figure 6: Reference objects allow comparison across subplots and can be more easily read at small scales than coordinate axes. In ggsubplot, users can add one of three types of reference objects to subplots by adding ref $=$ ref_box () , ref $=$ ref_hline(), or ref $=$ ref_vline() to geom_subplot and geom_subplot2d calls.

of the reference object can vary to provide additional information about a subplot. For example, the fill, color, and transparency of a reference object can display group level information about the data in a subplot. The ggsubplot reference parameter allows users to set these aesthetics with the functions ref_box, ref_vline and ref_hline, see Figure 6. By default, ref_box displays with a grey background and white border. This matches the color scheme of ggplot2's default background, while still delineating the dimensions of the subplot. Reference objects provide a quick way to compare across subplots. However, if users require a precise judgement they should still plot the subplot in its own graph with a pair of axes.

\section{References}

Wickham, H. (2010), "A layered grammar of graphics," Journal of Computational and Graphical Statistics, 19, 3-28.

- (2011), "The split-apply-combine strategy for data analysis," Journal of Statistical Software, $40,1-29$.

Wilkinson, L. and Wills, G. (2005), The Grammar of Graphics, Springer Verlag. 